Check for updates

Cite this: RSC Adv., 2017, 7, 24690

\section{Novel $\mathrm{Fe}_{3} \mathrm{O}_{4} \mathrm{aGNF}_{\mathrm{GSiO}}$ nanocapsules fabricated through the combination of an in situ formation method and $\mathrm{SiO}_{2}$ coating process for magnetic resonance imaging $\dagger$}

\author{
Changyong Lu, ${ }^{\text {ab }}$ Stefania Sandoval, ${ }^{\mathrm{b}}$ Teresa Puig, ${ }^{\mathrm{b}}$ Xavier Obradors, ${ }^{\mathrm{b}}$ \\ Gerard Tobias, (D) ${ }^{\mathrm{b}}$ Josep Ros ${ }^{\mathrm{a}}$ and Susagna Ricart (DD ${ }^{\mathrm{b}}$
}

\begin{abstract}
An in situ approach for the synthesis of $\mathrm{Fe}_{3} \mathrm{O}_{4}$ nanoparticles combined with a $\mathrm{SiO}_{2}$ coating process was employed to prepare $\mathrm{Fe}_{3} \mathrm{O}_{4} \mathrm{aGNF}_{\mathrm{aSiO}}$ nanocapsules. Graphitised nanofibres (GNF) were initially filled with iron(III) acetylacetonate, and used as a precursor for the synthesis of ultrasmall $\mathrm{Fe}_{3} \mathrm{O}_{4}$ nanoparticles ( $4.6 \mathrm{~nm}$ in diameter) inside the cavities of $\mathrm{GNF}$ ( $\mathrm{Fe}_{3} \mathrm{O}_{4} \mathrm{QGNF}$ ) with a high density. By using a silica coating process, $\mathrm{Fe}_{3} \mathrm{O}_{4} @ \mathrm{GNF}\left(\mathrm{aSiO}_{2}\right.$ nanocapsules were obtained. The presence of the silica shell not only prevented leakage of the nanoparticles from inside the GNF but also protected the magnetite nanoparticles from dissolution, even in harsh acidic conditions. Furthermore, the silica coating resulted in an increased dispersability of the nanocomposites in water. Magnetic resonance imaging (MRI) studies indicate relatively high $r_{2}^{*}$ relaxivities for $\mathrm{Fe}_{3} \mathrm{O}_{4} \mathrm{QGNF}$ nanocomposites and $\mathrm{Fe}_{3} \mathrm{O}_{4} \mathrm{aGNF} \mathrm{aSiO}_{2}$ nanocapsules revealing the potential application of these hybrid materials for bioimaging. Therefore, the coating of filled GNF with silica is as an excellent strategy for the protection of encapsulated payloads.
\end{abstract}

Received 10th April 2017 Accepted 28th April 2017

DOI: $10.1039 / \mathrm{c} 7 \mathrm{ra0} 4080 f$

rsc.li/rsc-advances a theoretical study by Pederson and Broughton who showed that fullerene tubules could draw molecules from liquid or vapor phase into their inner channels by capillary force. ${ }^{6}$ After that, a wide variety of both organic and inorganic materials have been encapsulated or even grown inside carbon nanotubes and carbon nanofibers forming hybrid structures. For example, metal halides, ${ }^{7}$ metallic nanowires, ${ }^{8}$ low dimensional silicabased materials, ${ }^{9}$ fluorescent polystyrene beads, ${ }^{10}$ and individual layers of graphene ${ }^{11}$ and related $2 \mathrm{D}$ materials ${ }^{12}$ have all been encapsulated inside CNTs. Due to their small inner diameter, although single-walled carbon nanotubes and multiwalled carbon nanotubes have been filled with a variety of nanomaterials, the amount of material that can be encapsulated is limited. On another side, hollow graphitised nanofibres (GNF) would be promising for encapsulating nanomaterials because of the relative large inner diameter. ${ }^{13}$ Andrei N. Khlobystov and his colleagues successfully synthesize $\mathrm{Au}$ nanoparticles inside $\mathrm{GNF}^{14,15}$ and assemble $\mathrm{Mn}_{3} \mathrm{O}_{4}$ nanoparticles inside $\mathrm{GNFs}^{16}$ making this kind of nanohybrids promising for the application in catalysis and spintronic devices. $\mathrm{Pt}$ and $\mathrm{RuO}_{2}$ nanoparticles can be synthesized and filled inside the nanofiber showing potential applications in electrocatalysis, charge storage and fuel cell research. ${ }^{17-19}$ Organic materials like fullerene-tagged [Cu-(salen)] catalysts and fullerene-free $\mathrm{Pd}(\mathrm{II})$ salen catalysts were immobilized inside GNF to form welldefined heterogeneous catalysts with excellent stability and recyclability. ${ }^{20,21}$ Recently, researchers also found out that Pt 
nanoparticles immobilized in shortened hollow graphitized carbon nanofibers could form an extremely stable electrocatalyst shown superior properties compared with commercial $\mathrm{Pt} / \mathrm{C}$ electrocatalyst. ${ }^{22}$ It is also worth to mention that GNF loaded with magnetic nanoparticles exhibit a large variety of current and potential applications in hyperthermia therapeutic and magnetic resonance imaging research. ${ }^{23}$

Several strategies have been employed to load the inner cavities of carbon nanotubes, ${ }^{24}$ but regardless of the method employed, bulk filling typically results in samples that contain non-encapsulated material external to the walls. Strategies to remove this external material include the use of reverse micelles ${ }^{25}$ and selective solvent mixtures, ${ }^{26}$ leading to samples of open-ended filled tubes clean of external material. In order to provide protection of the encapsulated cargo several strategies have been explored to seal or cork the ends. The first report on the formation of permanently sealed "carbon nanocapsules" (filled carbon nanotubes with closed ends) involved high thermal annealing of single-walled carbon nanotubes, which results in the spontaneous end-closing forming covalent bonds. ${ }^{27}$ Several other strategies have followed to block, at least partially, the open ends of the CNTs and GNF, including the use of fullerenes, ${ }^{28,29}$ gold,$^{30,31}$ silica, ${ }^{32}$ and a mixture of metal oxide nanoparticles. ${ }^{33}$ Removable corks have also been designed for the development of controlled release systems. ${ }^{30,31,34}$ It is also possible to coat the whole surface of nanohybrids with an inert protective shell. Following the work by Rao et al., where a protective layer of carbon was deposited on the surface of previously filled carbon nanotubes, ${ }^{35}$ Hampel et al. have recently used this strategy to prevent the release of toxic gadolinium from CNTs. ${ }^{36}$ The carbon layer has been deposited by thermal treatment of the filled CNTs using either acetylene or benzene vapor. While there are several papers about sealing the ends of CNTs, the work about closing the ends of GNF still remains challenging due to their large diameter; only a few reports talk about using graphene or metal particles to cork the ends of GNF. ${ }^{37,38}$

Among the different applications of filled carbon nanostructures, their use in the biomedical field for both imaging and therapy is taking the lead. ${ }^{39}$ In this area, the encapsulation of magnetic materials is of interest for the development of contrast agents for magnetic resonance imaging (MRI) and as therapeutic materials by hyperthermia. ${ }^{23}$ By capillary action, GNF and CNTs can be loaded with pre-formed magnetic iron oxide nanoparticles. ${ }^{\mathbf{4 0 - 4 2}}$ It is also possible to use GNF and CNTs as templates for the in situ synthesis of iron oxide nanoparticles in their interior..$^{33,43,44}$ Despite several authors have reported on the magnetic properties of the resulting hybrids, their performance as MRI test has been barely investigated. ${ }^{33}$

Here we combined the in situ synthesis of $\mathrm{Fe}_{3} \mathrm{O}_{4}$ nanoparticles inside the cavities of GNF and the $\mathrm{SiO}_{2}$ coating process to fabricate novel $\mathrm{Fe}_{3} \mathrm{O}_{4} @ G N F @ \mathrm{SiO}_{2}$ nanocapsules. The hybrid materials present a complete filling of the inner GNF cavities with magnetite nanoparticles, which in turn are sealed with a protective $\mathrm{SiO}_{2}$ coating. Due to the presence of the silica layer, the obtained nanocapsules still present a good magnetic response even after an acid treatment. It is also worth to mention that the formation of the silica layer not only prevents the leakage of the nanoparticles but also renders the hybrid material water dispersible, a key aspect for their use in biomedia. Since silica is a biocompatible and FDA approved material, these novel $\mathrm{Fe}_{3} \mathrm{O}_{4} @ \mathrm{GNF} @ \mathrm{SiO}_{2}$ nanocapsules arise as a promising material for biotechnology research. So far, the MRI tests in the present work have already indicated good $r_{2}^{*}$ relaxivities revealing the potential application in bio-imaging test. It is worth to mention here that with the combination of biocompatible $\mathrm{SiO}_{2}$ surface and good $r_{2}^{*}$ relaxivities, this kind of nanocapsules may have promising application in magnetic therapy, magnetic manipulation, drug delivery and disease diagnose.

\section{Results and discussion}

Functional GNF with magnetic nanoparticles could combine the features of magnetic nanoparticles and GNF, resulting in novel physical and chemical properties. The role of different experimental conditions on the in situ synthesis of magnetic nanoparticles was initially assessed. Two samples of acid treated GNF were impregnated with the iron precursor, and independently submitted to thermal activation by either traditional heating with a heating plate or via a controlled microwave irradiation process. Both protocols lead to the formation of iron oxide nanoparticles inside GNF (Fig. 1).

Detailed analysis by transmission electron microscopy (TEM) of the sample prepared by traditional thermal activation at $200{ }^{\circ} \mathrm{C}$ (Fig. 1a and b), reveals the complete filling of the inner cavities of the GNF with very small nanoparticles (pristine GNF in Fig. S1†). The presence of some nanoparticles decorating the external walls is also visible. A high-resolution image is presented in the Fig. $1 \mathrm{~b}$ to better appreciate the size of the nanoparticles, which have an average diameter of $4.6 \pm 0.5 \mathrm{~nm}$. When the $\mathrm{Fe}_{3} \mathrm{O}_{4}$ nanoparticles are synthesized in benzyl alcohol without presence of GNF, larger (7-8 nm) nanoparticles are formed, ${ }^{45}$ indicating the role of the GNF as templates during the in situ growth. It is worth to mention that, the amount of $\mathrm{Fe}(\mathrm{acac})_{3}$ used in the synthesis of $\mathrm{Fe}_{3} \mathrm{O}_{4} @ G N F$ nanocomposites is very important to make the GNF fully filled with nanoparticles. For instance, when the concentration of initial reactant $\mathrm{Fe}(\mathrm{acac})_{3}$ is reduced from 1 to $0.1 \mathrm{mmol}$, the obtained GNF presented lower density of nanoparticles inside (see Fig. S3†).

Since magnetic nanoparticles can be prepared by microwave treatment and GNF are highly microwave absorbing materials, the synthesis of the nanoparticles was next done under microwave irradiation. This allows us to compare the effect of microwave irradiation on the formation of the hybrids. TEM analysis also confirms the presence of iron oxide nanoparticles inside the cavities of the GNF (Fig. S4†). It is noteworthy that a partial filling of the nanotubes is observed, with a bubble-type morphology, which might come from the rapid heating process by microwave irradiation.

Finally, the effect of surface functionalization of GNF on the in situ formation of metal oxide nanoparticles was investigated. Steam cleaned GNF were used to prepare iron oxide nanoparticles inside the cavities by traditional thermal activation. 

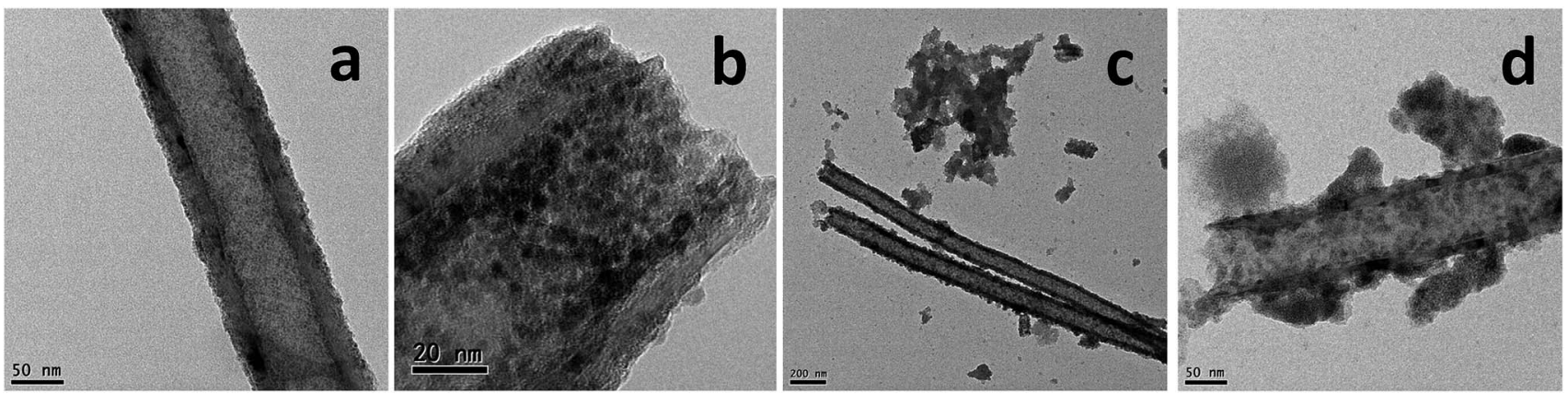

Fig. 1 (a) TEM image of $\mathrm{Fe}_{3} \mathrm{O}_{4}$ (aGNF nanocomposites prepared using acid treated GNF by traditional thermal activation, (b) high magnification image of $\mathrm{Fe}_{3} \mathrm{O}_{4}$ @GNF nanocomposites prepared using acid treated GNF; (c and d) $\mathrm{Fe}_{3} \mathrm{O}_{4}$ @GNF nanocomposites prepared using surface clean GNF (without nitric acid treatment) by traditional thermal activation.

TEM analysis (Fig. 1c and d) indicates the presence of iron oxide nanoparticles, but the loading density is not as high as when using acid treated GNF (Fig. 1a and b). This could be explained by the low dispersibility of the steam cleaned GNF in ethanol which might hinder their complete filling with the iron precursor. Furthermore, aggregated nanoparticles are also observed in Fig. 1e and $f$ which might account for the decomposition of the non-encapsulated $\mathrm{Fe}(\mathrm{acac})_{3}$ precursor still remaining outside the steam cleaned GNF, although leakage of nanoparticles during the washing process cannot be disregarded. Therefore, a preliminary conclusion can be made according to the discussion above based on TEM analysis: iron oxide filled GNF can be synthesized with a high filling density by using acid treated GNF and traditional thermal activation. Thus, this sample was employed for all the studies presented from now on. Finally, it is worth to mention that, although $\mathrm{Fe}_{3} \mathrm{O}_{4} @ \mathrm{GNF}$ nanocomposites are prepared with lower loading density via microwave irradiation than with the thermal approach, further investigation is needed since it is possible to explore more in the relation with the confinement effect and high microwave effect coming from the carbon structure.

$\mathrm{X}$-ray diffraction (XRD) analysis was next performed on the sample of filled tubes to determine the nature and structure of the synthesized nanoparticles. The most intense peaks observed in the XRD pattern in Fig. 2 could be assigned to

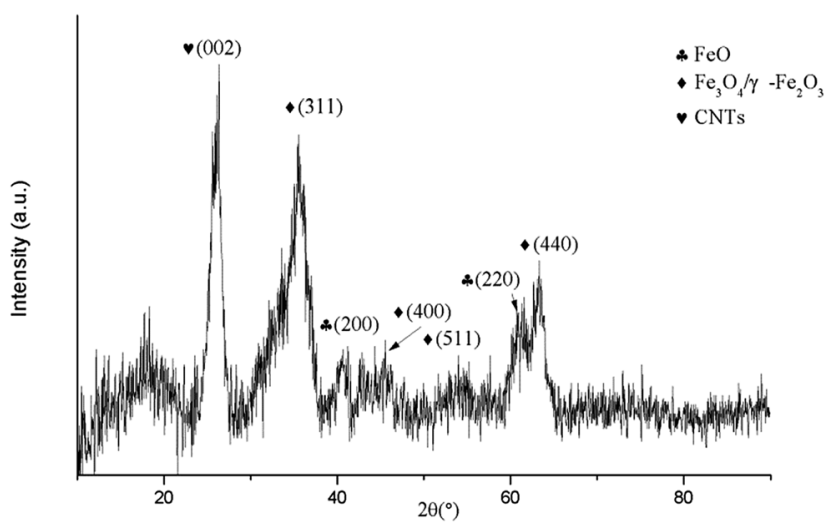

Fig. 2 X-ray diffraction pattern of $\mathrm{Fe}_{3} \mathrm{O}_{4} \mathrm{QGNF}$ nanocomposites prepared using acid treated GNF by traditional thermal activation. carbon (JCPDS no. 41-1487) and to the presence of either magnetite (JCPDS no. 19-0629) or maghemite (JCPDS no. 391346). The existence of iron(II) oxide (JCPDS no. 89-7100) is also detected. According to our previous studies on the synthesis of magnetic nanoparticles in the absence of GNF, the employed protocol should lead to the formation of magnetite nanoparticles $\left(\mathrm{Fe}_{3} \mathrm{O}_{4}\right){ }^{45}$ The presence of small nanoparticles is responsible for the relatively low signal to noise ratio in the XRD pattern. No peaks of iron(III) acetylacetonate which was used as the reactant are observed suggesting a complete decomposition by the thermal treatment. The wide peaks in the pattern can be attributed to the small size of the nanoparticles and may be explained by the confinement effect of GNF.

Magnetic measurements were carried out on the $\mathrm{Fe}_{3} \mathrm{O}_{4} @-$ GNF nanocomposites at $5 \mathrm{~K}$ (Fig. 3). The magnetization saturation value steadily increases as a function of the applied magnetic field indicating that the nanoparticles in the GNF are magnetically active, reaching a saturation magnetization of 34.5 emu $^{-1}$. The curve also displays a very small hysteresis featured by coercive field $\left(H_{\mathrm{c}}\right)$ of 280 Oe indicating a ferrimagnetic behavior. The magnetic behavior of the $\mathrm{Fe}_{3} \mathrm{O}_{4} @ \mathrm{GNF}$

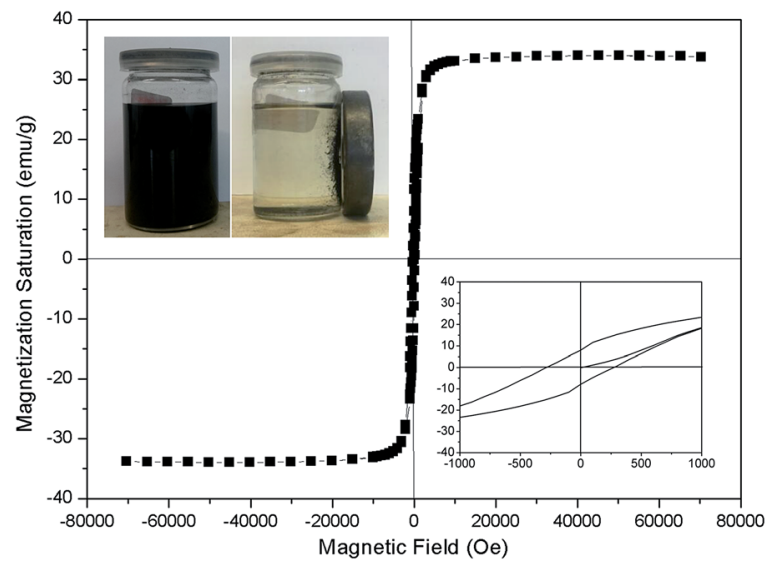

Fig. 3 Hysteresis loops at $5 \mathrm{~K}$ of $\mathrm{Fe}_{3} \mathrm{O}_{4} \mathrm{QGNF}$ nanocomposites prepared using acid treated GNF by traditional thermal activation. Inset top-left: photos of the nanocomposites dispersed in ethanol before and after (10 $\mathrm{min})$ contact with an external magnet. Inset bottom-right: detail of magnetization at the low magnetic field region. 
composites can be already appreciated by placing a magnet close to a dispersion of the nanocomposite in ethanol (see inset in Fig. 3). The image demonstrates that the nanocomposite can be easily manipulated by an external magnet as all the nanocomposite was separated from the solution within just few seconds. The fact that the hybrids can be easily separated in this manner is a clear advantage for their manipulation when compared to conventional filtration or centrifugation processes.

The amount of nanoparticles present in the nanocomposite was quantified by thermogravimetric analysis (TGA) under oxygen (Fig. S2 $\dagger$ ). The residue obtained after the complete combustion of the GNF at $600{ }^{\circ} \mathrm{C}$ corresponds to the $\mathrm{Fe}_{3} \mathrm{O}_{4}$ nanoparticles present in the sample which get oxidized to $\mathrm{Fe}_{2} \mathrm{O}_{3}$ during the TGA analysis. The amount of magnetite nanoparticles was calculated to $39.9 \mathrm{wt} \%$ based on the assumption that all the nanoparticles are magnetite. The theoretical value of the saturation magnetization, assuming that all the nanoparticles are pure $\mathrm{Fe}_{3} \mathrm{O}_{4}$ and taking into account that bulk $\mathrm{Fe}_{3} \mathrm{O}_{4}$ has a saturation magnetization of $92 \mathrm{emu} \mathrm{g}^{-1}$ is about $36.7 \mathrm{emu}$ $\mathrm{g}^{-1}$ which was slightly larger than the magnetization saturation data and can account for the presence of a small amount of $\mathrm{FeO}$ or be due to the fact that the magnetization saturation values of nanoparticles is usually smaller than that of the bulk. ${ }^{46}$
To complete the characterization of the nanocomposites, scanning transmission electron microscopy (STEM) and energy electron loss spectroscopy (EELS) were employed to determine the distribution of the $\mathrm{Fe}_{3} \mathrm{O}_{4}$ nanoparticles in the nanocomposite. Both STEM and EELS in Fig. 4 confirms the presence of a large amount of nanoparticles filling the inner cavities of the nanotubes. A small fraction of magnetite nanoparticles is also visible outside the GNF which correspond to the iron signals around $30 \mathrm{~nm}$ and $100 \mathrm{~nm}$.

The stability of the hybrid materials was next assessed by washing the as-synthesized $\mathrm{Fe}_{3} \mathrm{O}_{4} @ G N F$ nanocomposites (Fig. 5a) with different washing times using the procedure described in the Experiment part. Whereas an initial washing of the sample does not seem to release the encapsulated nanoparticles (Fig. 5b), when the samples were washed 2 times or more, leakage of the nanoparticles from the cavity was observed (Fig. 5c and d). As shown in the TEM images, the released nanoparticles tend to form big aggregates outside the GNF. The leakage of the magnetite nanoparticles hinders the application of the developed hybrids as contrast agents in the biomedical field since it would result in a misleading interpretation on the localization of the hybrid by in vivo imaging. To overcome this limitation, we propose a sealing strategy to shield materials inside GNF that consists on the formation of nanocapsules by
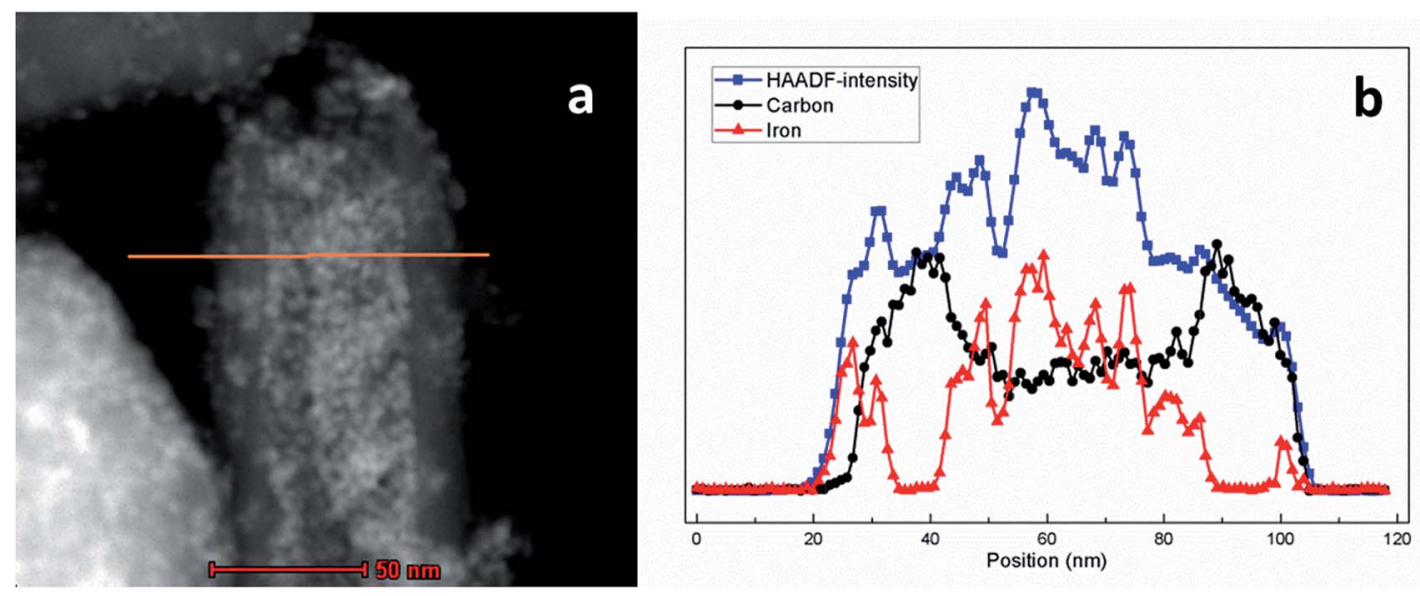

Fig. 4 (a) STEM and (b) high-angle annular dark-field imaging (HAADF), EELS analysis of $\mathrm{Fe}_{3} \mathrm{O}_{4} @$ GNF nanocomposites prepared using acid treated CNTs by traditional thermal activation.

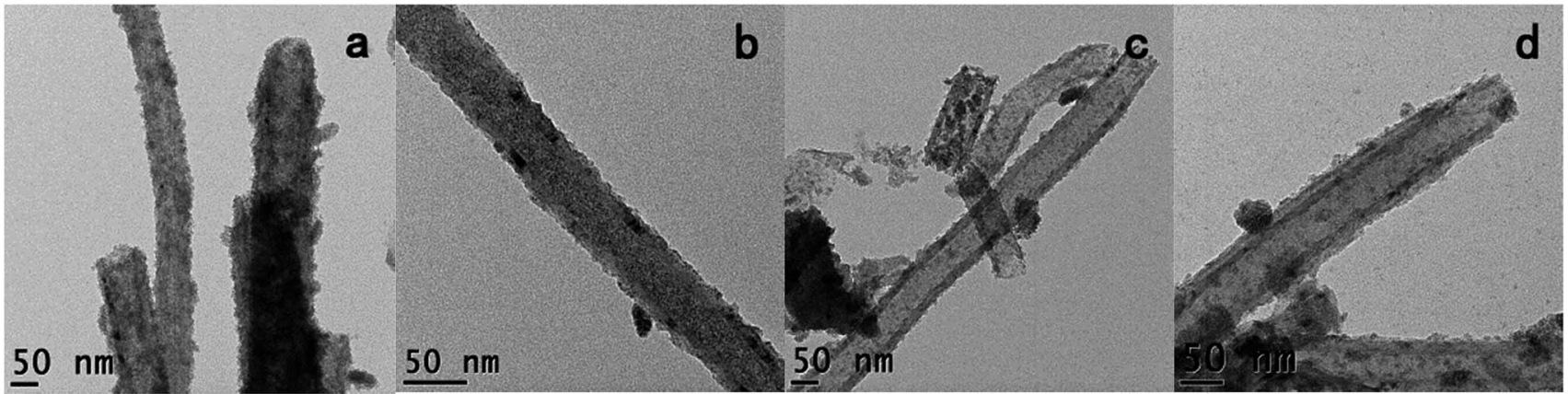

Fig. 5 TEM images of $\mathrm{Fe}_{3} \mathrm{O}_{4}$ (CCNF samples prepared using acid treated CNF by traditional thermal activation after different times of washing, (a) without wash, (b) 1 time, (c) 2 times and (d) 3 times. 


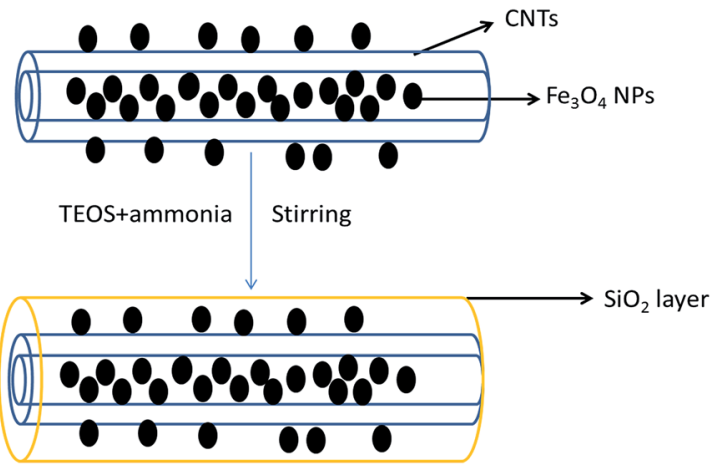

Scheme 1 Illustration of the formation of silica nanocapsules. Schematic representation of $\mathrm{SiO}_{2}$ coating of $\mathrm{Fe}_{3} \mathrm{O}_{4} @ \mathrm{GNF}$ nanocomposites

silica coating the entire surface of GNF. In the Scheme 1 it is shown a representation of the proposed approach.

The surface of the filled GNF was coated with a $\mathrm{SiO}_{2}$ shell via a straightforward method. The synthesized $\mathrm{Fe}_{3} \mathrm{O}_{4} @$ @GNF nanocomposites were simply mixed with TEOS (tetraethyl orthosilicate) in basic conditions which lead to a hydrolysis reaction. The FT-IR (Fig. 6) spectrum of the $\mathrm{Fe}_{3} \mathrm{O}_{4} @ \mathrm{GNF} @ \mathrm{SiO}_{2}$ nanocapsules shows absorption peaks at $795 \mathrm{~cm}^{-1}, 942 \mathrm{~cm}^{-1}, 1048 \mathrm{~cm}^{-1}, 1150$ $\mathrm{cm}^{-1}$ and $1205 \mathrm{~cm}^{-1}$ attributed to the formation of the $\mathrm{SiO}_{2}$ layer ${ }^{47}$ on the surface of $\mathrm{Fe}_{3} \mathrm{O}_{4} @ \mathrm{GNF}$ composites. A HR-TEM

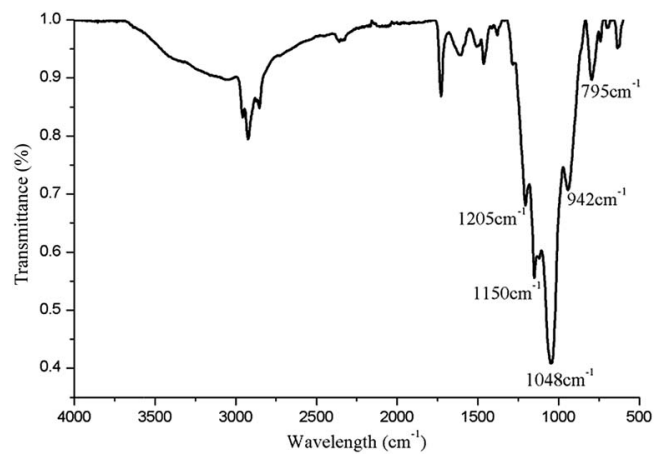

Fig. 6 FT-IR spectra curve of $\mathrm{Fe}_{3} \mathrm{O}_{4} \mathrm{OGNF} \mathrm{aSiO}_{2}$ nanocomposites. image of the prepared $\mathrm{Fe}_{3} \mathrm{O}_{4} @ \mathrm{GNF} @ \mathrm{SiO}_{2}$ nanocapsules is presented in Fig. 8a. In order to test the sealing capabilities of the developed $\mathrm{SiO}_{2}$-based nanocapsules, the $\mathrm{Fe}_{3} \mathrm{O}_{4} @ \mathrm{GNF} @ \mathrm{SiO}_{2}$ were washed with an excessive amount of an aqueous solution of $\mathrm{HCl}$. It is noteworthy that the cavities of the tubes were still fully filled with $\mathrm{Fe}_{3} \mathrm{O}_{4}$ nanoparticles (Fig. $7 \mathrm{~b}$ and c), providing direct evidence that the $\mathrm{SiO}_{2}$ coating is an efficient way to prevent the dissolution and leakage of the encapsulated compounds.

The magnetic behavior of $\mathrm{Fe}_{3} \mathrm{O}_{4} @ \mathrm{GNF} @ \mathrm{SiO}_{2}$ nanocapsules was also confirmed by applying an external magnet to a dispersion of the sample. As it can be seen in Fig. 8b, the acid washed $\mathrm{Fe}_{3}-$ $\mathrm{O}_{4} @ \mathrm{GNF} @ \mathrm{SiO}_{2}$ nanocapsules can be easily separated by the external magnetic field. It is worth to mention that the $\mathrm{SiO}_{2}$ shell not only confers robustness to the prepared hybrid but also enhances its dispersity. Although the $\mathrm{Fe}_{3} \mathrm{O}_{4} @ G N F$ nanocomposites can also be separated with the external magnet (Fig. 8a), some precipitation is observed on the bottom of the vial which was also visible in the inset image of Fig. 3 indicates the relatively low dispersion in polar solvents. This simple analysis already suggests that the coating of $\mathrm{SiO}_{2}$ improves the dispersion of the developed nanocomposites which is crucial for their application in biomedia.

The formation of the $\mathrm{Fe}_{3} \mathrm{O}_{4} @ \mathrm{GNF} @ \mathrm{SiO}_{2}$ nanocapsules (acid washed) was further confirmed by an EDS line profile

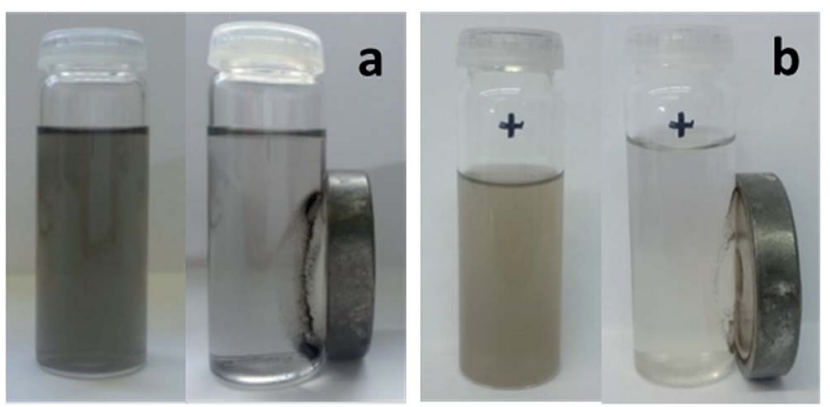

Fig. 8 Photos of (a) $\mathrm{Fe}_{3} \mathrm{O}_{4}$ aGNF nanocomposites and (b) $\mathrm{Fe}_{3} \mathrm{O}_{4}$ (a$\mathrm{CNFCSiO}_{2}$ nanocapsules (after being washed with an $\mathrm{HCl}$ aqueous solution), before and after ( $10 \mathrm{~min}$ ) applied external magnet. The same iron concentration was employed for the samples, which were dispersed in ethanol.

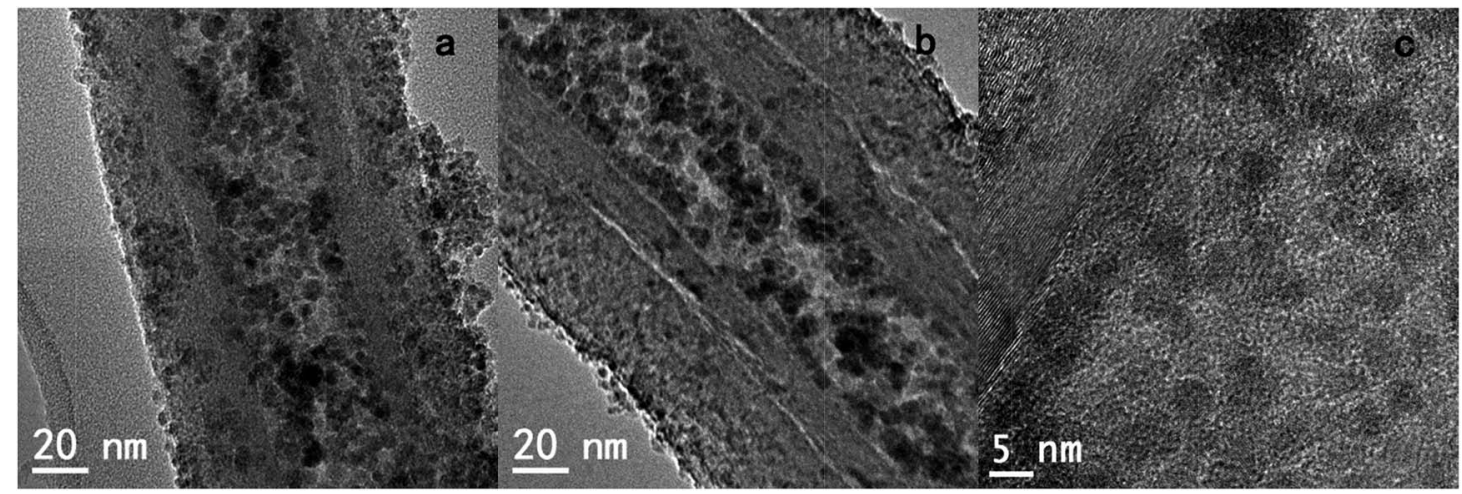

Fig. $7 \mathrm{HR}-\mathrm{TEM}$ images of $\mathrm{Fe}_{3} \mathrm{O}_{4} \mathrm{aGNF}_{\mathrm{aSiO}}$ nanocapsules (a) before and (b) after washing with an $\mathrm{HCl}$ aqueous solution; (c) high magnification image of $\mathrm{Fe}_{3} \mathrm{O}_{4} \mathrm{QGNF}\left(\mathrm{CSiO}_{2}\right.$ nanocapsules after being washed with an $\mathrm{HCl}$ aqueous solution. 

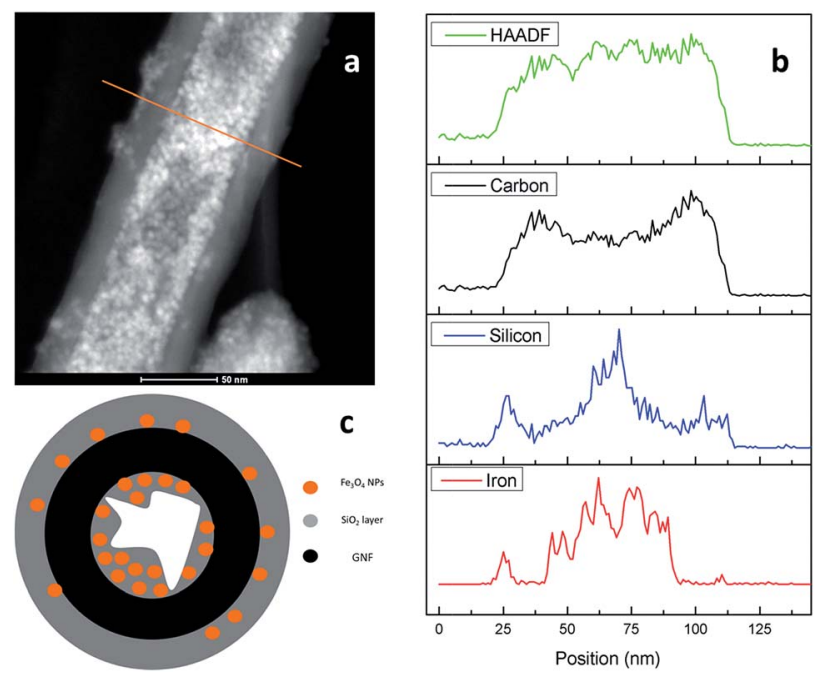

Fig. 9 (a) STEM and (b) EDS line profile analysis of the middle part of $\mathrm{Fe}_{3} \mathrm{O}_{4} \mathrm{aGNF} \mathrm{aSiO}_{2}$ nanocapsule after being washed by an $\mathrm{HCl}$ aqueous solution. (c) Schematic model of the $\mathrm{Fe}_{3} \mathrm{O}_{4} \mathrm{aGNF}\left(\mathrm{SiO}_{2}\right.$ nanocapsules

(Fig. 9a and b). First of all, a high Fe atomic ratio was observed inside the GNF confirmed the presence of $\mathrm{Fe}_{3} \mathrm{O}_{4}$ nanoparticles after the acid washing treatment suggesting the formation of protecting layer. As expected, Si signal could be observed on the external walls of the GNF, indicating the presence of the $\mathrm{SiO}_{2}$ shell and the shell thickness is around $20 \mathrm{~nm}$ identified by the TEM studies (Fig. 8a and b). At the same time a high Si atomic ratio is visible in the same position where the atomic content of iron is also high (especially inside the GNF) which suggests that the $\mathrm{SiO}_{2}$ was formed on the surface of the $\mathrm{Fe}_{3} \mathrm{O}_{4}$ nanoparticles. It is also observed that the GNF are not fully filled with nanoparticles, indicating a partial leakage of $\mathrm{Fe}_{3} \mathrm{O}_{4}$ nanoparticles during the hydrolysis of TEOS. A schematic model of the developed nanocapsules is presented in Fig. 9c, suggesting that the $\mathrm{SiO}_{2}$ layer was not only formed on the external surface of the GNF but also inside the cavity. ICP-MS analysis of the $\mathrm{Fe}_{3} \mathrm{O}_{4} @ G N F @ \mathrm{SiO}_{2}$ nanocapsules (Table $\mathrm{S} 1 \dagger$ ) after the complete digestion of the sample reveals the presence of $6.75 \mathrm{wt} \%$ of magnetite nanoparticles and a $65 \mathrm{wt} \%$ of silica coating. The relative fraction of GNF would then be $28.25 \mathrm{wt} \%$. In agreement with TEM analysis, ICP-MS confirms the partial leakage of magnetite nanoparticles during the hydrolysis of TEOS. TGA analysis of the $\mathrm{Fe}_{3} \mathrm{O}_{4} @ G N F @ \mathrm{SiO}_{2}$ nanocapsules (Fig. S5 $\dagger$ ) reveals a solid residue of $c a .85 \mathrm{wt} \%$ (at $900{ }^{\circ} \mathrm{C}$ ). The TGA residue should correspond to $\mathrm{Fe}_{2} \mathrm{O}_{3}$ (from $\mathrm{Fe}_{3} \mathrm{O}_{4}$ ) and the silica coating, since the complete combustion of the GNF would be expected at this temperature. The high solid residue collected after the TGA indicates that some GNF could be protected by the silica coating from oxidation during the TGA.

Since magnetic nanocomposites show great potential as MRI contrast agents, ${ }^{\mathbf{4 8 , 4 9}}$ relaxivity measurements of $\mathrm{Fe}_{3} \mathrm{O}_{4} @ \mathrm{GNF}$ nanocomposites and $\mathrm{Fe}_{3} \mathrm{O}_{4} @ \mathrm{GNF} @ \mathrm{SiO}_{2}$ nanocomposites were performed to evaluate their potential application as MRI contrast agents. The transverse $R_{2}^{*}$ relaxation rates of $\mathrm{Fe}_{3} \mathrm{O}_{4}$ @GNF nanocomposites and $\mathrm{Fe}_{3} \mathrm{O}_{4} @ G N F @ \mathrm{SiO}_{2}$ nanocomposites dispersed in 1.5\% agar and 1\% Pluronic F127 solution at various Fe concentration were measured at 7 T. Fig. 10 shows the phantom MR imaging of the samples. The relaxation times of these two nanocomposites were plotted versus the iron concentration, revealing their potential as negative contrast agents. The $r_{2}^{*}$ relaxivity of $\mathrm{Fe}_{3} \mathrm{O}_{4} @ G N F$ nanocomposites was calculated as $230.8 \mathrm{~s}^{-1} \mathrm{mM}^{-1}$ which is $70 \%$ of the value of commercial Endorem contrast agent $\left(325 \mathrm{~s}^{-1} \mathrm{mM}^{-1}\right)$. The high viscosity of the agar solution makes the test media difficult to go inside the GNF, which can actually result in the determination of lower $r_{2}^{*}$. An increase on the $r_{2}^{*}$ relaxivity to $252 \mathrm{~s}^{-1} \mathrm{mM}^{-1}$ was observed for the sample coated with silica $\left(\mathrm{Fe}_{3} \mathrm{O}_{4} @ G N F @ \mathrm{SiO}_{2}\right)$ making it an appealing probe for MRI test. In the meantime, the biocompatible $\mathrm{SiO}_{2}$ surface of these nanocapsules will make them more dispersible in bio-media and easy to be functionalized with biomolecules and other drugs indicating the promising application as a multifunctional tools in biomedicine research.

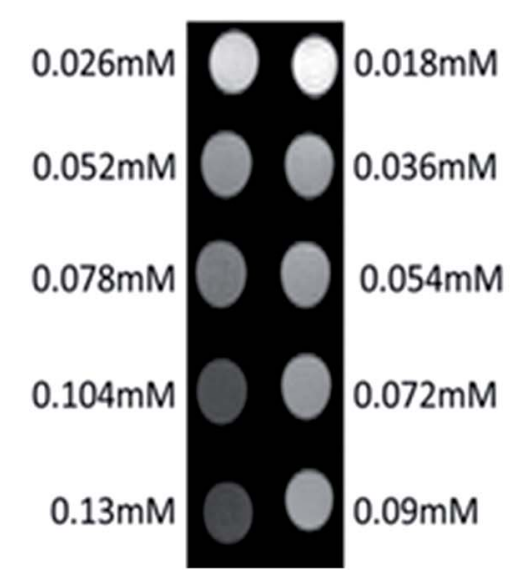

a

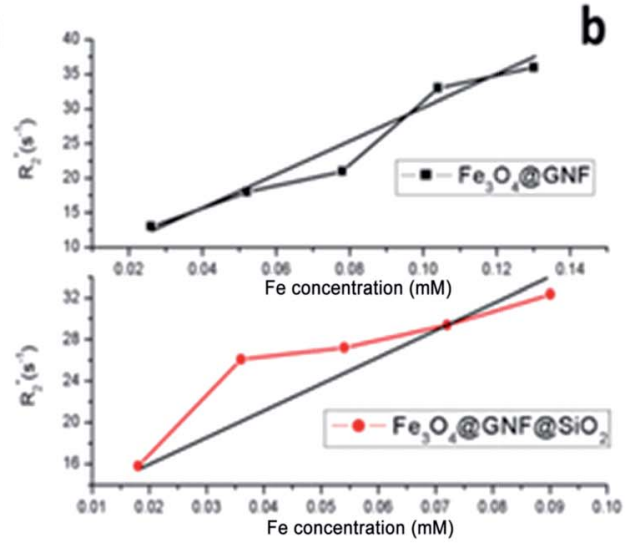

Fig. 10 (a) Phantom MR imaging of $\mathrm{Fe}_{3} \mathrm{O}_{4} \mathrm{QGNF}$ nanocomposites (left) and $\mathrm{Fe}_{3} \mathrm{O}_{4} \mathrm{QGNF}_{\mathrm{aSiO}}$ nanocapsules (right), (b) $R_{2}^{*}$ relaxation time analysis as a function of Fe concentration. 


\section{Conclusions}

In the present study, we have developed in situ synthesis of ultra-small iron oxide nanoparticles $(4.6 \pm 0.5 \mathrm{~nm})$ inside the cavities of GNF with a high filling density (39.9 wt\% of $\mathrm{Fe}_{3} \mathrm{O}_{4}$ ). The magnetic properties of the prepared nanocomposites allow an easy manipulation and separation of the sample with an external magnet. Furthermore, we reported on the use of silica as a sealing agent to obtain novel $\mathrm{Fe}_{3} \mathrm{O}_{4} @ G N F @ \mathrm{SiO}_{2}$ nanocapsules. The completely sealed $\mathrm{SiO}_{2}$ layer prevented leakage of the nanoparticles and protected them from dissolution in harsh conditions $(\mathrm{HCl})$. The developed silica nanocapsule not only offers protection of the encapsulated payload but also increases the dispersability of the hybrids, a key advantage for their application in the biomedical field. MRI studies of these two nanocomposites, namely $\mathrm{Fe}_{3} \mathrm{O}_{4} @ \mathrm{GNF}$ and $\mathrm{Fe}_{3} \mathrm{O}_{4} @ \mathrm{GNF} @ \mathrm{SiO}_{2}$, confirm their potential as negative contrast agents. Therefore, the prepared $\mathrm{Fe}_{3} \mathrm{O}_{4} @ \mathrm{GNF} @ \mathrm{SiO}_{2}$ nanocapsules combine excellent properties for their use as biomedical probes, including robustness, dispersability and contrast enhancement. We believe that the silica coating strategy presented herein for the containment of iron oxide nanoparticles inside GNF will also allow protection of other confined materials and decoration of biomolecules. In summary, the magnetic $\mathrm{Fe}_{3} \mathrm{O}_{4} @ G N F @ \mathrm{SiO}_{2}$ nanocomposites with biocompatible surface would have potential application as a multifunctional tool in biomedicine research and their applications in other fields are still need further study.

\section{Acknowledgements}

We acknowledge financial support from Spanish Ministry of Economy and Competitiveness through the "Severo Ochoa" Programme for Centres of Excellence in R\&D (SEV-2015-0496), CONSOLIDER Excellence Network (MAT2015-68994-REDC), COACHSUPENERGY project (MAT2014-51778-C2-1-R, cofinanced by the European Regional Development Fund) and CHALENG (MAT2014-53500-R). We also thank support from the European Union for EUROTAPES project (FP7-NMP-Large-2011280432) and from the Catalan Government with 2014-SGR-753 and Xarmae. We also acknowledge financial support through PIE 201660E013 (CSIC) and Chinese Scholarship Council (CSC).

\section{Notes and references}

1 S. Iijima, nature, 1991, 354, 56-58.

2 Y. Chen, X. Li, K. Park, J. Song, J. Hong, L. Zhou, Y.-W. Mai, H. Huang and J. B. Goodenough, J. Am. Chem. Soc., 2013, 135, 16280-16283.

3 D. Eder, Chem. Rev., 2010, 110, 1348-1385.

4 B. K. Balan and S. Kurungot, Inorg. Chem., 2012, 51, 97669774.

5 C. J. Shearer, A. Cherevan and D. Eder, Adv. Mater., 2014, 26, 2295-2318.

6 M. R. Pederson and J. Q. Broughton, Phys. Rev. Lett., 1992, 69, 2689.
7 J. Sloan, A. I. Kirkland, J. L. Hutchison and M. L. H. Green, Chem. Commun., 2002, 1319-1332, DOI: 10.1039/b200537a.

8 L. Guan, K. Suenaga, S. Okubo, T. Okazaki and S. Iijima, J. Am. Chem. Soc., 2008, 130, 2162-2163.

9 Z. Liu, S.-K. Joung, T. Okazaki, K. Suenaga, Y. Hagiwara, T. Ohsuna, K. Kuroda and S. Iijima, ACS Nano, 2009, 3, 1160-1166.

10 B. M. Kim, S. Qian and H. H. Bau, Nano Lett., 2005, 5, 873878.

11 A. Chuvilin, E. Bichoutskaia, M. C. Gimenez-Lopez, T. W. Chamberlain, G. A. Rance, N. Kuganathan, J. Biskupek, U. Kaiser and A. N. Khlobystov, Nat. Mater., 2011, 10, 687-692.

12 L. Cabana, B. Ballesteros, E. Batista, C. Magén, R. Arenal, J. Oró-Solé, R. Rurali and G. Tobias, Adv. Mater., 2014, 26, 2016-2021.

13 E. Castillejos, P.-J. Debouttière, L. Roiban, A. Solhy, V. Martinez, Y. Kihn, O. Ersen, K. Philippot, B. Chaudret and P. Serp, Angew. Chem., 2009, 121, 2567-2571.

14 A. La Torre, M. d. C. Giménez-López, M. W. Fay, G. A. Rance, W. A. Solomonsz, T. W. Chamberlain, P. D. Brown and A. N. Khlobystov, ACS Nano, 2012, 6, 2000-2007.

15 A. La Torre, G. A. Rance, J. El Harfi, J. Li, D. J. Irvine, P. D. Brown and A. N. Khlobystov, Nanoscale, 2010, 2, 1006-1010.

16 M. d. C. Gimenez-Lopez, A. La Torre, M. W. Fay, P. D. Brown and A. N. Khlobystov, Angew. Chem., Int. Ed., 2013, 52, 20512054.

17 B. K. Balan, S. M. Unni and S. Kurungot, J. Phys. Chem. C, 2009, 113, 17572-17578.

18 B. Cornelio, A. R. Saunders, W. A. Solomonsz, M. LaronzeCochard, A. Fontana, J. Sapi, A. N. Khlobystov and G. A. Rance, J. Mater. Chem. A, 2015, 3, 3918-3927.

19 R. Vellacheri, V. K. Pillai and S. Kurungot, Nanoscale, 2012, 4, 890-896.

20 M. A. Lebedeva, T. W. Chamberlain, M. Schröder and A. N. Khlobystov, Chem. Mater., 2014, 26, 6461-6466.

21 M. A. Lebedeva, T. W. Chamberlain, A. Thomas, B. E. Thomas, C. T. Stoppiello, E. Volkova, M. Suyetin and A. N. Khlobystov, Nanoscale, 2016, 8, 11727-11737.

22 M. del Carmen Gimenez-Lopez, A. Kurtoglu, D. A. Walsh and A. N. Khlobystov, Adv. Mater., 2016, 28, 9103-9108.

23 A. Stopin, F. Pineux, R. Marega and D. Bonifazi, Chem.-Eur. J., 2015, 21, 9288-9301.

24 M. Monthioux and E. Flahaut, Mater. Sci. Eng., C, 2007, 27, 1096-1101.

25 J. Cook, J. Sloan, R. J. R. Heesom, J. Hammer and M. L. H. Green, Chem. Commun., 1996, 2673-2674, DOI: 10.1039/cc9960002673.

26 Q. Fu, W. Gisela and D.-s. Su, New Carbon Mater., 2008, 23, 17-20.

27 L. Shao, G. Tobias, Y. Huh and M. L. H. Green, Carbon, 2006, 44, 2855-2858.

28 L. Shao, T.-W. Lin, G. Tobias and M. L. H. Green, Chem. Commun., 2008, 2164-2166, DOI: 10.1039/b800881g.

29 Y. Ren and G. Pastorin, Adv. Mater., 2008, 20, 2031-2036. 
30 J. Li, S. Q. Yap, S. L. Yoong, T. R. Nayak, G. W. Chandra, W. H. Ang, T. Panczyk, S. Ramaprabhu, S. K. Vashist, F.-S. Sheu, A. Tan and G. Pastorin, Carbon, 2012, 50, 16251634.

31 J. Li, S. L. Yoong, W. J. Goh, B. Czarny, Z. Yang, K. Poddar, M. M. Dykas, A. Patra, T. Venkatesan, T. Panczyk, C. Lee and G. Pastorin, Int. J. Nanomed., 2015, 10, 7425-7744.

$32 \mathrm{X}$. Chen, H. Chen, C. Tripisciano, A. Jedrzejewska, M. H. Rümmeli, R. Klingeler, R. J. Kalenczuk, P. K. Chu and E. Borowiak-Palen, Chem.-Eur. J., 2011, 17, 4454-4459.

33 X. Liu, I. Marangon, G. Melinte, C. Wilhelm, C. c. MénardMoyon, B. P. Pichon, O. Ersen, K. Aubertin, W. Baaziz and C. Pham-Huu, ACS Nano, 2014, 8, 11290-11304.

34 P. Luksirikul, B. Ballesteros, G. Tobias, M. G. Moloney and M. L. Green, Carbon, 2010, 48, 1912-1917.

35 B. C. Satishkumar, A. Govindaraj, J. Mofokeng, G. N. Subbanna and C. N. R. Rao, J. Phys. B: At., Mol. Opt. Phys., 1996, 29, 4925.

36 E. Fidiani, P. M. F. J. Costa, A. U. B. Wolter, D. Maier, B. Buechner and S. Hampel, J. Phys. Chem. C, 2013, 117, 16725-16733.

37 J. Shin, K. Park, W.-H. Ryu, J.-W. Jung and I.-D. Kim, Nanoscale, 2014, 6, 12718-12726.

38 Y. Shen, L. Li, K. Xiao and J. Xi, ACS Sustainable Chem. Eng., 2016, 4, 2351-2358.
39 M. Martincic and G. Tobias, Expert Opin. Drug Delivery, 2015, 12, 563-581.

40 G. Korneva, H. Ye, Y. Gogotsi, D. Halverson, G. Friedman, J.-C. Bradley and K. G. Kornev, Nano Lett., 2005, 5, 879-884.

41 S. Kopyl, V. Bystrov, I. Bdikin, M. Maiorov and A. C. Sousa, J. Mater. Chem. C, 2013, 1, 2860-2866.

42 S. Kopyl, A. Timopheev, V. Bystrov, I. Bdikin, B. Teixeira, E. Maevskij, N. Sobolev and A. Sousa, J. Magn. Magn. Mater., 2014, 358, 44-49.

43 W. Baaziz, X. Liu, I. Florea, S. Begin-Colin, B. P. Pichon, C. Ulhaq, O. Ersen, M. Soria-Sánchez, S. Zafeiratos and I. Janowska, J. Mater. Chem. A, 2013, 1, 13853-13861.

44 W. Chen, X. Pan and X. Bao, J. Am. Chem. Soc., 2007, 129, 7421-7426.

45 E. Solano, L. Perez-Mirabet, F. Martinez-Julian, R. Guzmán, J. Arbiol, T. Puig, X. Obradors, R. Yañez, A. Pomar and S. Ricart, J. Nanopart. Res., 2012, 14, 1-15.

46 B. Xavier and L. Amílcar, J. Phys. D: Appl. Phys., 2002, 35, R15. 47 C. Lu, T. Puig, X. Obradors, S. Ricart and J. Ros, RSC Adv., 2016, 6, 88762-88769.

48 Q. L. Vuong, J. F. Berret, J. Fresnais, Y. Gossuin and O. Sandre, Adv. Healthcare Mater., 2012, 1, 502-512.

49 L. Sandiford, A. Phinikaridou, A. Protti, L. K. Meszaros, X. Cui, Y. Yan, G. Frodsham, P. A. Williamson, N. Gaddum and R. M. Botnar, ACS Nano, 2012, 7, 500-512. 